\title{
Evidence for two-band magnetotransport in half-metallic chromium dioxide
}

\author{
S. M. Watts, S. Wirth, and S. von Molnár \\ MARTECH, Florida State University, Tallahassee, Florida 32306-4351
}

A. Barry and J. M. D. Coey

Department of Physics, Trinity College, Dublin 2, Ireland

(Received 23 February 1999; revised manuscript received 20 December 1999)

\begin{abstract}
Magnetotransport measurements were made on patterned, (110) oriented $\mathrm{CrO}_{2}$ thin films grown by the high-pressure, thermal decomposition of $\mathrm{CrO}_{3}$ onto rutile substrates. The low-temperature Hall effect exhibits a sign reversal from positive to negative as the magnetic field is increased above $1 \mathrm{~T}$, which may be interpreted within a simple two-band model as indicating the presence of highly mobile $\left(\mu_{h}=0.25 \mathrm{~m}^{2} / \mathrm{V} \mathrm{s}\right)$ holes as well as a much larger number of less mobile electrons $(n=0.4$ electrons $/ \mathrm{Cr})$. Between 50 and $100 \mathrm{~K}$, the field at which the sign reversal occurs rapidly increases and a contribution from the anomalous Hall effect becomes significant, while the large, positive transverse magnetoresistance (MR) observed at low temperatures changes over to a predominantly negative MR. These changes correlate with a thermally activated dependence in the resistivity of the form $T^{2} e^{-\Delta / T}$ with $\Delta \approx 80 \mathrm{~K}$, reflecting the lack of temperature dependence in the resistivity at low temperatures and a $T^{2}$ behavior above $100 \mathrm{~K}$. The high mobilities at low temperature which result in the observed positive MR reflect the suppression of spin-flip scattering expected for a half-metallic system. However, the changes in magnetotransport above the temperature $\Delta$ must be due to the onset of spin-flip scattering, even though $k_{B} \Delta$ is much less than the expected energy gap in the minority spin density of states. The significance of $\Delta$ is discussed in terms of recent models for another half-metallic system, the perovskite manganites, and the possible formation of "shadow bands."
\end{abstract}

\section{INTRODUCTION}

Chromium dioxide has long been a technologically important magnetic material, for as a powder it adheres well to media suitable for use in the magnetic-recording industry. However, it is not a stable $\mathrm{Cr}-\mathrm{O}$ phase under normal conditions, and special methods are needed to fabricate the material — such as high-pressure, thermal decomposition of higher $\mathrm{Cr}$ oxides. Under such conditions, $\mathrm{CrO}_{2}$ crystallizes as microscale needles. Thus not much has been known about the electronic structure of $\mathrm{CrO}_{2}$ as it was difficult to make crystals large enough for transport measurements. There is one exception in the early work on $\mathrm{CrO}_{2}$ by a group at Dupont, ${ }^{1}$ and a group at General Electric pioneered the growth of thin epitaxial films on rutile substrates. ${ }^{2}$ The measurements in both cases showed indisputably metallic conductivity, with the resistivity varying as $T^{2}$ at low temperatures. ${ }^{3}$ This established $\mathrm{CrO}_{2}$ as the only known ferromagnetic, metallic binary oxide. Along with a phenomenological, molecular-orbital model proposed by Goodenough ${ }^{4}$ that provided an explanation for the metallic and magnetic nature of $\mathrm{CrO}_{2}$, these works constituted the basis of the knowledge of electronic transport in $\mathrm{CrO}_{2}$ for over two decades.

Interest in the unusual properties of transition-metal oxides has prompted a number of band-structure calculations on $\mathrm{CrO}_{2}$ over the past few years. ${ }^{5-8}$ One was by Schwartz, ${ }^{5}$ who predicted that $\mathrm{CrO}_{2}$ would be half-metallic: the majority spin has a metallic band structure, while the minority spin has a semiconductorlike gap. His results can generally be said to agree with the Goodenough model. A notable difference is that the narrow, half full $\pi^{*}$ conduction band from the Goodenough model is split into two bands in this (and all subsequent) calculations. Thus in the latter case the Fermi energy would fall into a local minimum in the density of states generated by these partially overlapping bands, possibly giving rise to both hole and electron conduction in the sense of a semimetal.

Spin-polarized transport has also been a topic of renewed interest since the discovery of giant magnetoresistance (GMR) in 1988. In a half-metallic ferromagnet, the conduction electrons should be completely spin polarized (at least well below $T_{C}$ ). This makes $\mathrm{CrO}_{2}$ a good candidate for use as a spin injector and has sparked the revival of $\mathrm{CrO}_{2}$ thinfilm growth techniques, including the original high-pressure, thermal decomposition method ${ }^{2}$ as well as a chemical vapor deposition (CVD) technique discovered in the late 1970s. ${ }^{9}$ A number of measurements, including Kerr effect, ${ }^{10}$ photoemission spectroscopy, ${ }^{11}$ Andreev reflection, ${ }^{12}$ infrared spectroscopy, ${ }^{13}$ Raman spectroscopy, ${ }^{14}$ and magnetotransport, ${ }^{15-18}$ have been recently published (to name a few).

For applications that involve moving spin-polarized carriers through an interface, it is essential to know what states are available near the Fermi energy and how these states evolve with temperature. The magnetotransport measurements described here should help address these issues and determine the potential usefulness of $\mathrm{CrO}_{2}$ in sensor or spinelectronic devices.

\section{EXPERIMENT}

$\mathrm{CrO}_{2}$ films were fabricated by the high-pressure thermal decomposition of $\mathrm{CrO}_{3}$ onto (110)-oriented single-crystal 


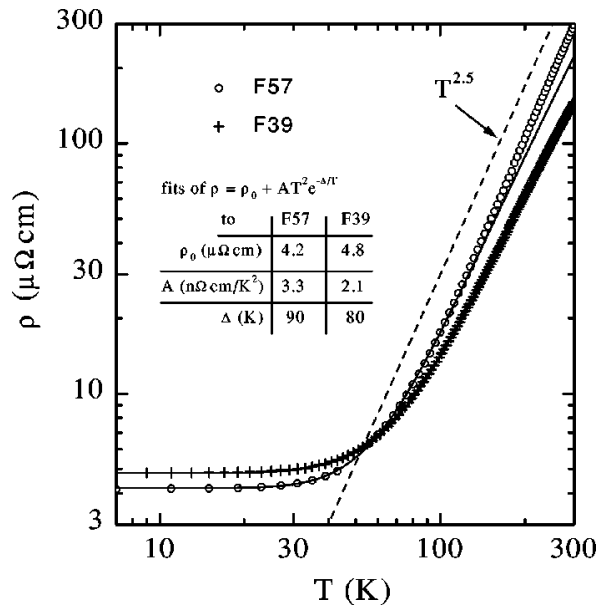

FIG. 1. Resistivity vs temperature taken during slow cools from room temperature. For sample F57 the current is parallel to the crystallographic $c$ axis, perpendicular for sample F39. The solid lines are from fits of Eq. (1) to the data; the fit parameters are given in the plot. The fit to F57 was limited to $150 \mathrm{~K}$.

rutile $\left(\mathrm{TiO}_{2}\right)$ substrates, as detailed previously. ${ }^{15}$ Such films have shown a record spin polarization of $90 \%,{ }^{12}$ and have been used in many recent studies. ${ }^{13,14,16}$ The films were textured and polycrystalline in character, exhibiting needlelike grains aligned in the film plane. Magnetic measurements indicate a Curie temperature of $395 \mathrm{~K}$ and an atomic magnetic moment near $2 \mu_{B}$, in accordance with published values. The approximately $0.5-\mu \mathrm{m}$-thick films were patterned into Hall bars using photolithography and wet chemical etching, and electrical leads were attached with indium solder or silver paint. Four-probe magnetoresistance (MR) and Hall-effect measurements were made simultaneously with a lowfrequency ac technique. For the Hall measurements, the voltage perpendicular to the current in a Hall cross geometry was collected for opposite directions of the magnetic field. The data could then be corrected for the magnetoresistive contribution due to misalignment of the voltage probes which is symmetric with field, to obtain the Hall voltage which is antisymmetric in field.

We will refer to two specific samples in what follows. All measurements made on the sample designated as F57 were conducted in an 8-T superconducting magnet system between 0.3 and $320 \mathrm{~K}$. Similar measurements were made for sample F39 at temperatures below $150 \mathrm{~K}$, and we established that the Hall effect and perpendicular MR were qualitatively the same for the two samples over this temperature range. All high-field measurements, which were conducted in an 18-T superconducting magnet system at the National High Magnetic Field Laboratory (NHMFL), were made on F39.

\section{RESULTS}

Resistivity traces taken during slow cools are shown in Fig. 1 for both samples. For F39 the current is perpendicular to the $c$ axis while for F57 the current is parallel to the $c$ axis. The dependence is rather flat at low temperatures, with residual resistivities between 4 and $5 \mu \Omega \mathrm{cm}$. The data may be fit by the phenomenological expression, ${ }^{16}$

$$
\rho=\rho_{0}+A T^{2} e^{-\Delta / T} .
$$

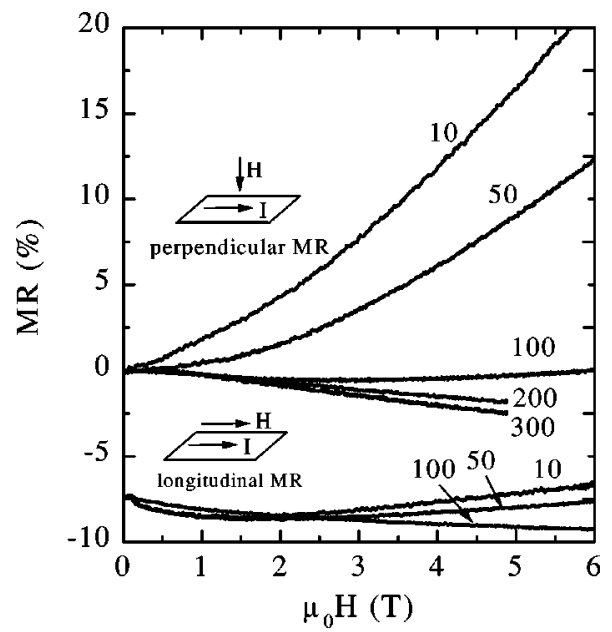

FIG. 2. Magnetoresistance curves at various temperatures for sample F57, with field either perpendicular to the film plane or in-plane and parallel to the current (indicated schematically in the plot). The longitudinal MR data is shifted down by $7 \%$ for clarity. $\mathrm{MR}=[\rho(H)-\rho(H=0)] / \rho(H=0)$.

A $T^{2}$ dependence could indicate either electron-electron or electron-magnon interactions. For the latter case, an exponential prefactor as in Eq. (1) would normally be attributed to a gap in the magnon spectrum. In Ref. 16 it is shown that there is no other evidence for a gap of only $\Delta \approx 80 \mathrm{~K}$, and the exponential prefactor was therefore associated with the inaccessibility of minority spin states for spin-flip scattering processes. A very similar form has been observed for the halfmetallic, colossal-MR manganites. ${ }^{19}$ For $\mathrm{CrO}_{2}$, the temperature $\Delta$ is clearly too low to be related directly to the band gap of order $1 \mathrm{eV}$ predicted by any of the bandstructure calculations.

Perpendicular (field perpendicular to the film plane) and longitudinal (field in-plane and parallel to the current) MR measurements on sample F57 are shown in Fig. 2. Transverse (field in-plane and perpendicular to the current) MR measurements for sample F39 are shown in Fig. 3. At low temperature, the perpendicular and transverse MR show a strong positive effect while the longitudinal MR is small and negative. The positive contribution to the MR falls off in magnitude as the temperature is increased, and the MR becomes dominated by a linear, negative contribution for $T$ $\gg \Delta$. These measurements are in good agreement with Ref. 18 , but are in contrast to the measurements of Ref. 17, in which a negative, saturating MR dominates at low temperatures for films which have a factor 20 larger residual resistivity than the films studied here and in Ref. 18.

Hall-effect measurements show a remarkable amount of variation with field and temperature. At high temperatures [Fig. 4(a)] the Hall signal resembles the magnetization and reflects the dominance of the second term in the phenomenological expression,

$$
\rho_{H}=R_{0} B+R_{S} \mu_{0} M
$$

where $R_{0}$ signifies the ordinary Hall coefficient and $R_{S}$ is the anomalous Hall coefficient. Above magnetic saturation the slope is positive linear, indicating that holelike carriers dominate the ordinary Hall coefficient. As the temperature is low- 


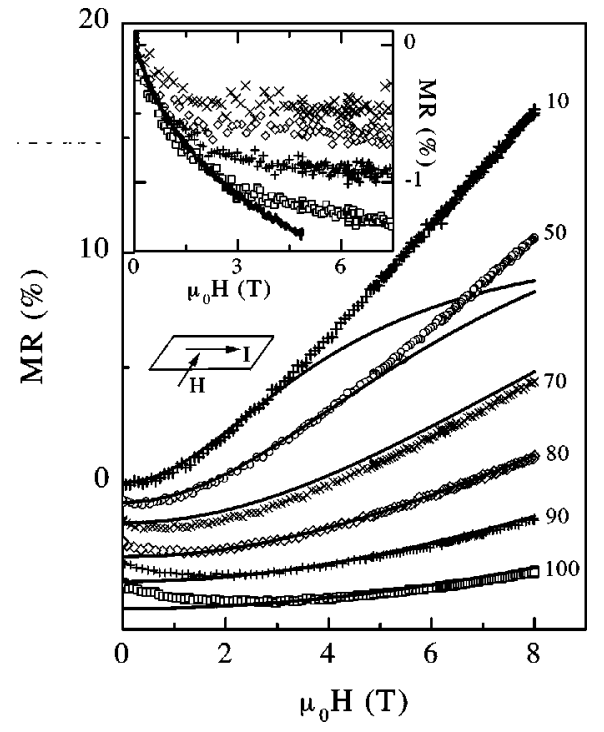

FIG. 3. Magnetoresistance traces for sample F39 at the indicated temperatures with field in-plane (parallel to $c$ ) and perpendicular to the current. For each isotherm above $10 \mathrm{~K}$ the data are shifted down by a constant amount for clarity. Solid lines represent fits of Eq. (8) to the data; above $70 \mathrm{~K}$ the fit curves are further shifted down relative to the data in order to offset the negative MR contribution to the data and demonstrate the high-field fit quality. The inset shows the difference between the data and the fit curve for temperatures $70 \mathrm{~K}$ and above. Raw MR data taken at $130 \mathrm{~K}$ are shown for comparison.

ered, the anomalous Hall coefficient rapidly decreases. At 10 and $50 \mathrm{~K}$ [Fig. 4(b)], the anomalous Hall effect is completely absent, yet the Hall resistance is observed to curve smoothly from a positive slope to a negative slope, and then to change

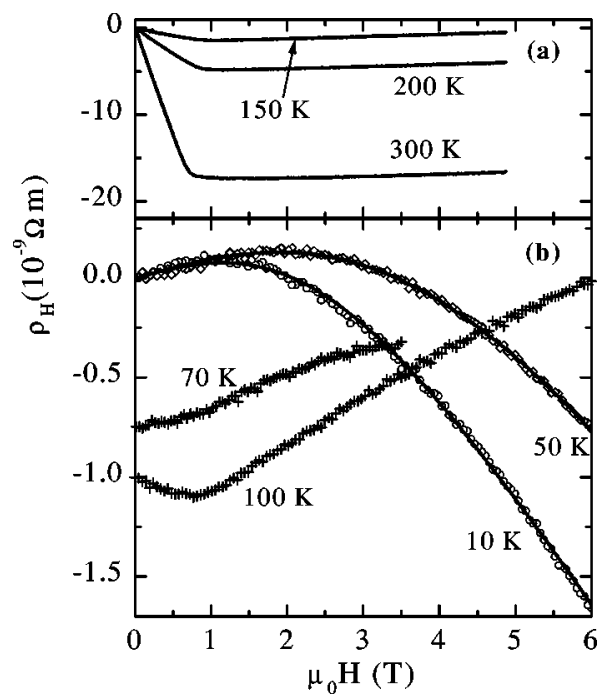

FIG. 4. Hall resistivity vs field for sample F57 at temperatures between 150 and $300 \mathrm{~K}$ (a), and between 10 and $100 \mathrm{~K}$ (b). The data in (a) are dominated by a large, negative anomalous Hall effect; a linear positive slope may be observed above the magnetic saturation field. The low-temperature data (b) show a sign reversal with field at 10 and $50 \mathrm{~K}$ and strong curvature above $50 \mathrm{~K}$ which is unrelated to the anomalous Hall effect. Solid lines are fits of Eq. (3) to the $10-$ and $50-\mathrm{K}$ data. The 70 and $100 \mathrm{~K}$ data are shifted down for clarity.

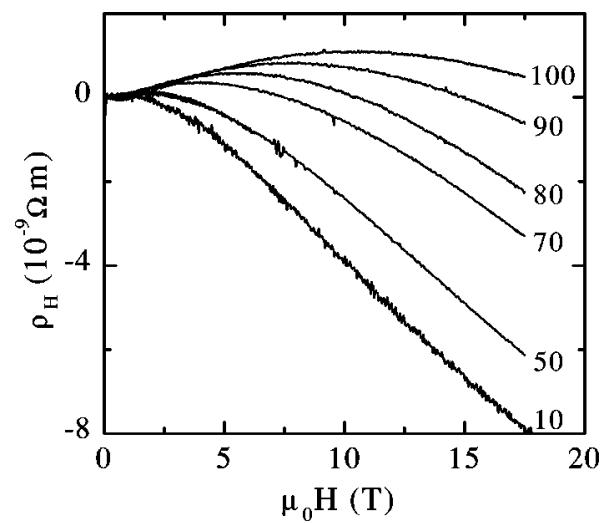

FIG. 5. Hall resistivity vs field for sample F39 at the indicated temperatures, with fields up to $18 \mathrm{~T}$. The sign reversal is observed up to $90 \mathrm{~K}$ and the trend continues at $100 \mathrm{~K}$. There is a small contribution from the anomalous Hall effect evident at low fields between 70 and $100 \mathrm{~K}$.

sign at a field $B_{0} . B_{0}$ is observed to increase smoothly as the temperature is raised, such that higher field measurements are necessary to determine its value at temperatures around $\Delta$ and above. Such measurements were made on sample F39 for temperatures between 10 and $100 \mathrm{~K}$, as shown in Fig. 5 . The sign reversal is observed to continue up to $100 \mathrm{~K}$, with $B_{0}$ directly measureable up to $90 \mathrm{~K}$.

\section{ANALYSIS}

\section{A. Hall effect}

A sign reversal in the ordinary Hall effect clearly indicates contributions from both hole and electronlike orbits on the Fermi surface. This can be due to field-induced changes in the nature of a particular orbit, as for magnetic breakdown in elemental $\mathrm{Cr},{ }^{20}$ and for intersheet field-dependent scattering in $\mathrm{Cd}^{21}$ These effects are normally accompanied by dramatic oscillations in the MR, which we do not observe here. Sign reversals with field are also observed in some of the simple metals, such as $\mathrm{Al}$ and $\mathrm{In},{ }^{22}$ for which a complete analysis involves integration over their known Fermi surfaces. ${ }^{23}$ Sign reversals with field are fairly common in semiconductors when heavy holes-light electrons are encountered, and can be understood with a simple two-band model. As a first approximation, the two-band model may also be applied in the simple metals (like Al and In, see Ref. 23) and alloys ${ }^{24}$ in order to understand the essential physics. We take this approach here, for lack of any measurement of the Fermi surface. Qualitatively, the field dependence that we find can be understood immediately with the two-band model in terms of the product $\omega_{c} \tau=\mu B$ particular to each band, where $\omega_{c}$ is the cyclotron frequency, $\tau$ is the relaxation time, and $\mu$ is the mobility. The positive slope in low fields reflects the dominance of highly mobile holes in the lowfield condition, where $\mu B \ll 1$ for both bands. Above $B_{0}$, the holes have moved into the high-field condition, $\mu B \gg 1$, and the negative slope reflects the dominance of the more numerous electrons.

To obtain more quantitative results, we may fit the data to the expression for the two-band Hall resistivity ${ }^{25}$ (ignoring the anomalous term for the moment): 


$$
\rho_{H}=\frac{\sigma_{h}^{2} R_{h}-\sigma_{e}^{2} R_{e}-\sigma_{h}^{2} \sigma_{e}^{2} R_{h} R_{e}\left(R_{h}-R_{e}\right) B^{2}}{\left(\sigma_{e}+\sigma_{h}\right)^{2}+\sigma_{e}^{2} \sigma_{h}^{2}\left(R_{h}-R_{e}\right)^{2} B^{2}} B,
$$

where $\sigma_{e}$ and $\sigma_{h}$ are the individual electron and hole band conductivities, respectively, and $R_{e}$ and $R_{h}$ are the respective Hall coefficients. All four parameters in this case are positive, as the signs have been explicitly incorporated into Eq. (3). This expression is general for two bands, with no assumptions made about the parameters. In the simplest case of spherical hole and electron Fermi surfaces, these parameters are field independent and $R_{e}=1 / n e$ and $R_{h}=1 / p e$, where $n$ and $p$ are the electron and hole carrier concentrations, respectively, and $e$ is the magnitude of the electronic charge. We begin our analysis with these simplifying assumptions. We note that Eq. (3) is of the form

$$
y \equiv \frac{\rho_{H}}{B}=\frac{b-c x}{1+a x},
$$

where $x \equiv B^{2}$ and $a, b$, and $c$ are functions of the band parameters. Finding the values of $a, b$, and $c$ will give us three equations in the four unknown band parameters. Thus in order to determine any of the band parameters, we must have another equation. Following Ref. 24, we use the zero-field resistivity to relate the two-band conductivities:

$$
1 / \rho \approx \sigma=\sigma_{e}+\sigma_{h} .
$$

(The approximation of $1 / \rho \approx \sigma$ is estimated to be accurate to about $1 \%$ - better than the absolute accuracy of the measurement of $\rho$.) Using the zero-field resistivity is consistent with the assumption of spherical Fermi surfaces.

We start with three parameter fits of Eq. (3) to the lowtemperature F57 data, having defined $\sigma_{e}$ in terms of $\sigma_{h}$ and $\rho$. The fits are shown in Fig. 4(b). We find that $R_{e} \ll R_{h}$, implying $n \gg p$ (by a factor of 500). The value for $R_{e}$ corresponds to 0.4 electrons/Cr. The Hall mobility for the $i$ th band $(i=e, h)$ is defined as

$$
\mu_{i}=\sigma_{i} R_{i} .
$$

At $10 \mathrm{~K}$, we find the hole mobility to be about $0.25 \mathrm{~m}^{2} / \mathrm{V} \mathrm{s}$. The electron mobility is a factor 20 smaller. The data indicate that the electrons are the dominant conduction carriers, in agreement with early thermopower measurements that showed a small and negative Seebeck coefficient. ${ }^{39}$

We now turn to the high-field data for sample F39 and use Eq. (4) for the fitting, since it provides a more transparent method for determining these parameters, as we will show. The data above $50 \mathrm{~K}$ were corrected for the anomalous Hall contribution; the values obtained for $R_{S}$ are discussed below. In Fig. 6 we plot the F39 data as $R_{0}$ vs $B^{2}$ on a semilog scale. Three features are noteworthy: the horizontal asymptote at low field, corresponding to the low-field limit of Eq. (4) in which $y \rightarrow b$; the $x$ intercept at the field $x_{0}=B_{0}^{2}$, where $y=0$ in Eq. (4) such that $b=c x_{0}$ and so $c=b / x_{0}$; and another horizontal asymptote (observable in the data for the lowest temperature only) at high fields, corresponding to the limit $y \rightarrow-c / a$. Thus for much of the data we can directly infer the values for $b$ and $c$ and, at the lowest temperatures, $a$.

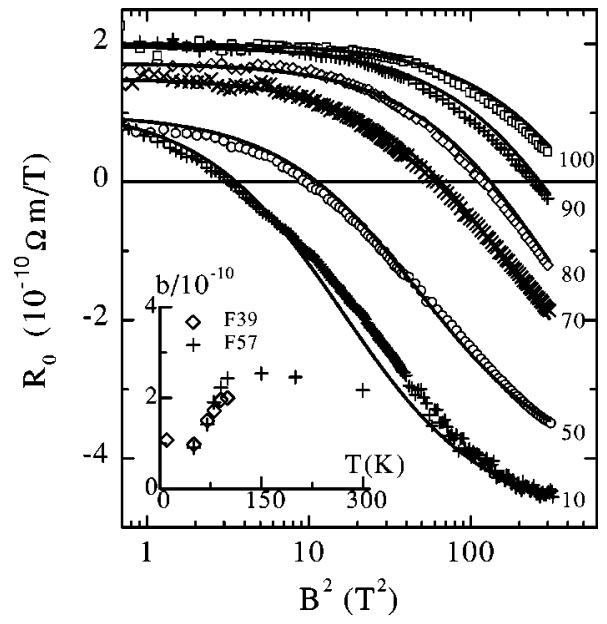

FIG. 6. The normal Hall coefficient $R_{0}$ vs $B^{2}$ for sample F39, where $R_{0}$ is defined in Eq. (2) and was obtained by subtracting out the anomalous Hall-effect contribution to the data shown in Fig. 5 (see text for details). Solid lines are fits of Eq. (4) to the data. The inset shows the dependence of $b$ (the zero-field value of $R_{0}$ ) vs temperature for both samples F39 and F57. In the two-band model, $b$ is given by Eq. (7b).

Fits of Eq. (4) to the F39 data are shown in Fig. 6. The fits were forced to match the low- and high-field asymptotic behavior at low temperatures, and do not match the intermediate field region well. In particular, the fit curves tend to overshoot the intercept $x_{0}$, resulting in a lower value for $c$. Since the low- and high-field extremes are better defined regimes (within the assumption that high fields do not affect the Hall coefficients), we have favored obtaining the best values of $b$ and $c / a$ over a fit procedure in which the overall difference between the fit curve and the data is minimized. This presents a problem for fitting the data at temperatures above 50 $\mathrm{K}$, as the high-field regime moves above $18 \mathrm{~T}$. The data may be fit very well with Eq. (4); however, forcing the fit curve to overshoot $x_{0}$ in a way that mimics the fits at low temperatures gives a feel for the range of possible $c / a$.

The parameters $a, b$, and $c$ may be transformed back to the band parameters with the following equations:

$$
\begin{gathered}
a=\left(\frac{\sigma_{e}}{\sigma} \mu_{h}\right)^{2}\left(1-\frac{R_{e}}{R_{h}}\right)^{2}, \\
b=\frac{\sigma_{h} \mu_{h}-\sigma_{e} \mu_{e}}{\sigma^{2}}=\left(\frac{\sigma_{h}}{\sigma}\right)^{2} R_{h}-\left(\frac{\sigma_{e}}{\sigma}\right)^{2} R_{e}, \\
c=\frac{R_{e} R_{h}}{R_{h}-R_{e}} a,
\end{gathered}
$$

where we have used Eqs. (5) and (6) to simplify the expressions. Because of the speculative nature of the hightemperature fits, it is instructive to first examine the temperature dependence of $b$ (Fig. 6, inset), including data up to 300 $\mathrm{K}$ from sample F57. Equation (7b) shows that $b$ is the difference between the Hall coefficients of each band weighted by their relative conductivities. If the carriers experience similar scattering rates and the carrier concentrations do not change significantly with temperature, then $b$ should not depend on temperature. This is indeed the case at 10 and $50 \mathrm{~K}$, 


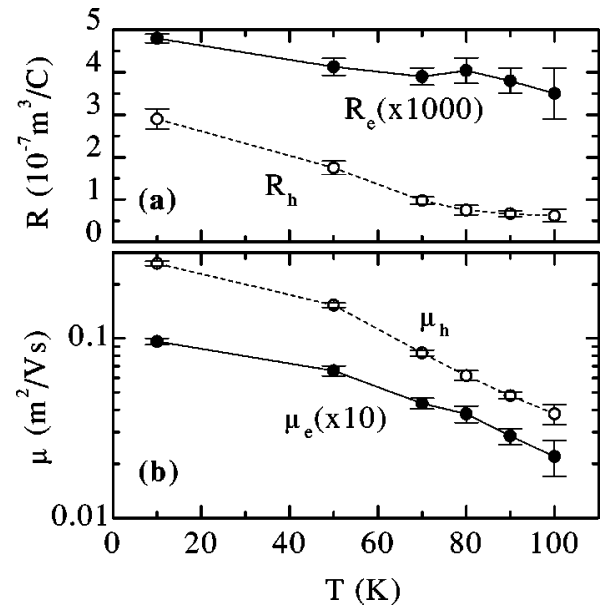

FIG. 7. Temperature dependence of the band parameters determined from the two-band analysis of the Hall-effect data. The top panel (a) shows the two Hall coefficients, with $R_{e}$ scaled by 1000 . The bottom panel (b) shows the two mobilities on a semilog scale, with $\mu_{e}$ scaled by 10 . Open circles are for holes, closed circles for electrons.

and above $100 \mathrm{~K}$. Between 50 and $100 \mathrm{~K}$, however, $b$ is observed to increase sharply, implying that the ratio $\sigma_{h} / \sigma_{e}$ is increasing in that temperature range.

The band parameters have been calculated from the experimental values for $b, c$, and $c / a$; their uncertainties were found by analytically propogating the large uncertainties associated with $c$ and $c / a$ through Eqs. (7). The resulting values for the Hall coefficients and mobilities are shown in Fig. 7. All of the values decrease with temperature, including a surprising factor 5 decrease in $R_{h}$ between 10 and $100 \mathrm{~K}$. The behavior of the mobilities is qualitatively similar for both bands. The inverse of the mobilities normalized to their $10-\mathrm{K}$ values are plotted together in Fig. 8. Both curves may be described with an $T^{2} e^{-\Delta / T}$ dependence (with $\Delta=80 \mathrm{~K}$ ). We note that $R_{h}$ and $\mu_{e}$ are quite sensitive to the value of the resistivity used in determining the parameters, although this was not included in the uncertainty calculation. It was observed that by decreasing the residual resistivity from 4.8 to $2 \mu \Omega \mathrm{cm}$, the low-temperature value of $R_{h}$ could be scaled down to roughly its high-temperature value while the low-

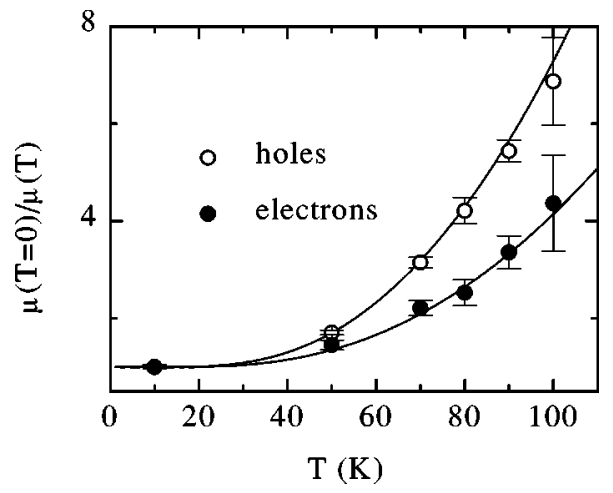

FIG. 8. Inverse mobilities, normalized to their $10-\mathrm{K}$ values, vs temperature. The inverse of the mobility is proportional to the scattering rate. The solid lines are fits of the function $f(T)=1$ $+A T^{2} e^{-\Delta / T}$, with $\Delta=80 \mathrm{~K}$.

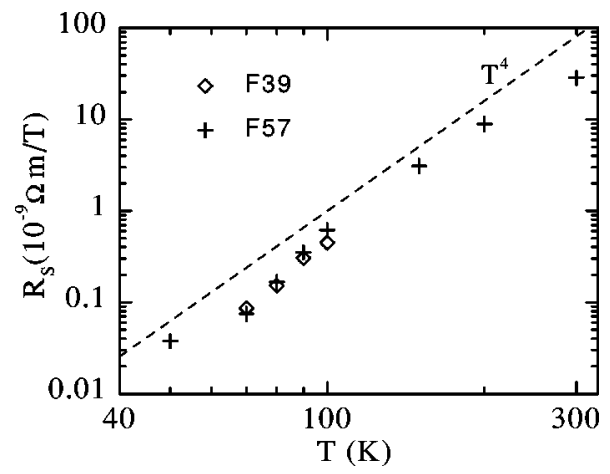

FIG. 9. The anomalous Hall coefficient vs temperature for samples F39 and F57. The dashed line is a $T^{4}$ curve shown for comparison.

temperature value of $\mu_{e}$ was increased. The qualitative temperature dependencies do not change.

The values for $R_{S}$ were found by extrapolating the linear portion of the Hall data at fields above technical saturation of the magnetization to zero field; the $y$ intercept is equal to $R_{S} \mu_{0} M_{S}$, where $M_{S}$ is the experimental value for the saturation magnetization. An upper bound for $R_{S}$ at $50 \mathrm{~K}$ for sample F57 is estimated to be $3.5 \times 10^{-11} \Omega \mathrm{m} / \mathrm{T}$ (no contribution is detectable for sample F39 at $50 \mathrm{~K}$ ). The values thus obtained for $R_{S}$ are plotted vs temperature for both samples on a log-log scale in Fig. 9. $R_{S}$ increases by three orders of magnitude between 50 and $300 \mathrm{~K}$; the dashed line in Fig. 9 shows a $T^{4}$ curve for comparison. This strongly suggests that the primary contribution to the anomalous Hall effect is from the side jump mechanism, ${ }^{26}$ which scales inversely with the mean free path. ${ }^{27}$ If the carrier polarization is proportional to the magnetization as inferred from the anomalous Hall data for some half-metallic Heusler alloys, ${ }^{28}$ then $R_{S}=\rho \Delta L / \lambda$ $\propto \rho^{2}$, where $\lambda$ is the mean free path and $\Delta L$ is the side jump distance (of order $1 \AA$ ); this suggests a $T^{4}$ dependence since $\rho \propto T^{2}$ above $100 \mathrm{~K}$. A more detailed analysis along the lines of Ref. 28 is made more difficult here by the presence of two bands, both of which may contribute to side jump, ${ }^{29}$ and whose relative contributions are changing with temperature, as indicated by the Hall measurements.

\section{B. Magnetoresistance}

A positive transverse MR is also characteristic of the twoband model. Furthermore, the longitudinal MR is zero in the simplest case of spherical Fermi surfaces. The expression for the two-band MR is ${ }^{30}$

$$
\frac{\Delta \rho}{\rho(0)} \equiv \frac{\rho(B)-\rho(B=0)}{\rho(B=0)}=\xi \frac{a x}{1+a x},
$$

where $a$ and $x$ are the same as in Eqs. (7a) and (4), and $\xi$ is related to the band parameters by

$$
\xi=\frac{\sigma_{h}}{\sigma_{e}} \frac{\left(1+\mu_{e} / \mu_{h}\right)^{2}}{\left(1-R_{e} / R_{h}\right)^{2}} .
$$

We note that Eq. (8) predicts a saturating quadratic behavior with field since $x=B^{2}$. The solid curves in Fig. 3 show the result of fitting Eq. (8) to the transverse MR data, using the values of $a$ obtained from the Hall fits and allowing $\xi$ to vary 
as a fitting parameter. These data were chosen since the field is in-plane and along the $c$ axis, thereby minimizing the influence of demagnetizing or anisotropy fields. At low temperatures, the MR data is well described by Eq. (8) up to 3-4 $\mathrm{T}$, above which the theoretical curve saturates while the experimental curve continues linearly. While not particularly well understood, this behavior is often found for many materials, the most notable example being the "free-electron", metal potassium. ${ }^{30}$ At higher temperatures, much better overall agreement is observed. We find $\xi$ to be approximately $1 / 10$ at $10 \mathrm{~K}$, increasing to approximately $1 / 4$ for $70 \mathrm{~K}$ and above-values which are larger by a factor of 2 to 3 than what one would obtain by evaluating $\xi$ with the band parameters shown in Fig. 7. This suggests that $\sigma_{h} / \sigma_{e}$ is somewhat larger than indicated by the Hall measurements, but not unreasonably so considering the simplicity of the model. Furthermore, it is evident at low fields for temperatures above $50 \mathrm{~K}$ that there is a negative MR contribution which increases in strength with increasing temperature. Subtracting the fit curve of Eq. (8) from the data gives some indication of the form of the negative MR, shown in the inset to Fig. 3.

\section{DISCUSSION}

The most important point to be made from the Hall-effect data is that both electrons and holes are responsible for charge transport in $\mathrm{CrO}_{2}$. The electrons make the dominant contribution to the conductivity, but the highly mobile holes determine low-field magnetotransport properties. The disparate characteristics of the two bands as determined by analysis of the low-temperature Hall effect suggest that the holes do not originate from overlapping $d$ bands, in the sense of a nearly compensated semimetal with both holes and electrons. The lack of significant difference in the temperature dependence of the scattering rates between the two bands demonstrated by Fig. 8 indicates that the effective mass of the holes is much less than that of the electrons and is more characteristic of a broad $p$-like band. This picture is most consistent with the band-structure calculation of Korotin et al., ${ }^{7}$ in which states of $\mathrm{O} 2 p$ character are observed to extend to $E_{F}$; these states are in addition to those due to the narrow, hybridized $p-d$ bands. Recent $\mathrm{x}$-ray-absorption spectra $^{31}$ and electron energy-loss spectra ${ }^{32}$ have shown good agreement with this band-structure calculation.

The high hole mobilities and low carrier number should give rise to Shubnikov-de Haas oscillations in the MR. No evidence for oscillations were observed in the MR, even up to $18 \mathrm{~T}$ at temperatures as low as $30 \mathrm{mK}$. Measurements of de Haas-van Alphen oscillations should be more conclusive, since it is known that Shubnikov-de Haas oscillations can be "shorted out" by the larger Fermi surfaces when both small and large surfaces are present. ${ }^{34}$

We do not wish to overinterpret the temperature evolution of the band parameters determined from the Hall-effect analysis, considering the uncertainties involved. We may say with confidence that the temperature dependence of the parameter $b$ (which corresponds to the initial, low-field slope of the Hall data) shows that the relative characteristics of the two bands are changing as the temperature is raised through $\Delta$, and specifically that $\sigma_{h} / \sigma_{e}$ is increasing. The analysis indicates that this is due to a decrease in $R_{h}$ (and hence an increase in $p$ and $\sigma_{h}$ ) rather than, say, different scattering rates. This is difficult to understand; however, it is interesting to note that a similar, inexplicable decrease in the Hall coefficient was observed for the half-metallic Heusler alloy $\mathrm{NiMnSb}$ for temperatures between 4 and $100 \mathrm{~K}$, with constant behavior above $100 \mathrm{~K} .{ }^{33}$ Since $b$ is constant above 100 $\mathrm{K}$, we suspect that $R_{h}$ is as well. There is also a measurable decrease in $R_{e}$ between 10 and $50 \mathrm{~K}$ as shown in Fig. 7, similar to that of PtMnSb from Ref. 33. That these features have some commonality in half-metallic ferromagnets and the likely association with an energy scale $\Delta$ warrants further investigation.

The evolution of spin disorder with temperature is a key problem in $\mathrm{CrO}_{2}$. Since the temperature $\Delta$ heralds not only a $T^{2}$ increase in the resistivity, but also the onset of the anomalous Hall effect and negative MR, it seems clearly related to a magnetic scattering process. In a half-metallic ferromagnet, magnetic scattering is limited by the inaccessibility of minority spin states. Scattering processes requiring a spin-flip are completely suppressed. For this reason, it was supposed ${ }^{35}$ that double magnon scattering-which does not induce a spin flip-would be the dominant scattering process at low temperatures in the manganites, giving $\rho \propto T^{4.5}$, and single magnon scattering would be supressed by a factor $e^{-E_{g} / k_{B} T}$, with $E_{g}$ the minority spin gap. All of the band-structure calculations indicate an $E_{g}$ of the order of $\mathrm{eV}$, and clearly $\Delta$ is on a much lower energy scale than this. But realistically, at finite temperatures there will be fluctuations in the localized $d$ moments, and on a length scale characterized by the magnon wavelength ${ }^{16}$ there will be a finite, effective density of minority states at $E_{F}$. Since the low-temperature magnetization of $\mathrm{CrO}_{2}$ follows the Bloch $T^{3 / 2}$ law, ${ }^{16,36}$ these minority band states may evolve as the "shadow bands" discussed in Ref. 37. Single magnon scattering in the manganites (which may have a small number of minority states at $E_{F}$ even at 0 $\mathrm{K})$ was studied by Jaime et al. ${ }^{19}$ They relate the suppression of the $T^{2}$ term in the resistivity to the minimum magnon energy $D q_{\min }$ ( $D$ is the spin-wave stiffness constant) which connects the majority and minority band Fermi momentum

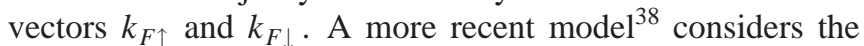
"nearly" half-metallic ferromagnet (namely, the manganites), and stipulates that the minority spin band at $\mathrm{E}_{F}$ does not conduct because of Anderson localization. The authors predict that $\rho \propto T^{2.5}$ above a characteristic temperature around $60 \mathrm{~K}$. All of these models probably hold some portion of the truth and are in many ways complimentary. They all predict essentially an onset of spin disorder scattering at temperatures of order $100 \mathrm{~K}$, above which the resistivity increases as $T^{2}$ or $T^{2.5}$. Both of the latter manganite models ${ }^{19,38}$ refer to a mechanism for the negative, nonsaturating MR. In Ref. 19 it is observed that the applied magnetic field opens a gap in the magnon spectrum, which would reduce magnon scattering. In Ref. 38 it is noted that an applied field will increase the Anderson localization length and thus reduce the magnonscattering matrix element.

We turn now to comparisons with previously published Hall data. In the earliest work, ${ }^{10}$ a Hall measurement at 300 $\mathrm{K}$ showed a large, negative anomalous Hall effect, with a negative slope above the saturation of the magnetization. We speculate that the linear, negative $\mathrm{MR}$ at this temperature 
may have contributed to this high-field slope. Recent measurements have been reported ${ }^{18}$ on films fabricated by the low-pressure CVD technique on (100) rutile. In magnetic fields up to $4 \mathrm{~T}$, the normal Hall slope measured on these films was positive linear at all temperatures. This is indeed a puzzling difference, since the anomalous Hall contribution as well as many of the other transport measurements in common are very similar, such as the resistivity vs temperature and some of the MR curves. To resolve these differences, we performed measurements on a similarly prepared film in magnetic fields up to $27 \mathrm{~T}$ at the NHMFL. The Hall effect is found to change sign in this sample, but at a much higher field. This measurement confirms that in both types of samples we have (a) two-band conduction, and (b) the dominant conduction carrier is the electron. Evidently there is a more delicate balance between the two-band parameters in the CVD film.

This difference in the observed hole band parameters could be due simply to the different orientations of the films: the CVD films are deposited on (100) rutile, whereas our films are on (110) rutile. Thus for the Hall measurements in the latter film, the field is directed along the $a$ - $a$ diagonal of the unit cell. For nonspherical Fermi surfaces, such as those predicted in Ref. 8, the Hall effect could be highly anisotropic. For instance, in iron a Hall curve similar to ours (but opposite in sign) is observed along one and only one specific orientation of the field relative to the crystallographic axes. ${ }^{40}$ If indeed the difference is not found to be a Fermi-surface orientation effect, then it must be related to sample dependent factors like crystallinity, strain, or oxygen content. The CVD-grown films are noted to be epitaxial and single crystalline, but highly strained. ${ }^{36}$

\section{CONCLUSIONS}

Low-temperature Hall-effect measurements on goodquality $\mathrm{CrO}_{2}$ films deposited on (110) rutile clearly exhibit contributions from both hole and electron bands. We have analyzed the data in terms of a simple two-band model and find evidence for a narrow, predominantly $d$ band containing 0.4 electrons/Cr as well as a broad $p$ band just crossing the Fermi level, yielding a very much smaller number of highly mobile holes. While the electrons are found to be the dominant conduction carrier, the contribution of the holes are important to low-field magnetotransport. The band parameters determined from the Hall-effect analysis give a reasonable account of the positive contribution to the transverse, field in-plane MR. As the temperature is increased through $\Delta$ $=80 \mathrm{~K}$, a crossover occurs from a well-behaved metallic regime to a "bad metal" regime, related to the onset of strong spin disorder scattering heralded by the appearance of a $T^{2}$ term in the resistivity, negative MR, and the anomalous Hall effect. The temperature $\Delta$ is probably related to the occurence of spin-flip scattering events due to local spin fluctuations that give a finite, effective density of minority spin states at $E_{F}$.

\section{ACKNOWLEDGMENTS}

We would like to thank A. Gupta for providing to us the CVD sample for our comparison measurements. Our gratitude also goes to R. J. Gambino for sharing with us unpublished data. The assistance of A. Anane has been of great benefit to this work, both through illuminating discussions and help with the measurements. Finally we thank P. Schlottman for his insight and interest in this work.

This research was sponsored by DARPA through the Office of Naval Research, ONR Grant Nos. N00014-96-1-0767 and N00014-99-1-1094. S.W. acknowledges support by the Humboldt Foundation, Germany.
${ }^{1}$ B.L. Chamberland, Mater. Res. Bull. 2, 827 (1967).

${ }^{2}$ D.S. Rodbell, J.M. Lommel, and R.C. DeVries, J. Phys. Soc. Jpn. 21, 2430 (1966).

${ }^{3}$ B.L. Chamberland, CRC Crit. Rev. Solid State Mater. Sci. 7, 1 (1977).

${ }^{4}$ J. B. Goodenough, in Progress of Solid State Chemistry, edited by H. Reiss (Permagon, Oxford, 1971), Vol. 5, Chap. 4, pp. 145399.

${ }^{5}$ K. Schwartz, J. Phys. F: Met. Phys. 16, L211 (1986).

${ }^{6}$ S.P. Lewis, P.B. Allen, and T. Sasaki, Phys. Rev. B 55, 10253 (1997).

${ }^{7}$ M.A. Korotin, V.I. Anisimov, D.I. Khomskii, and G.A. Sawatzky, Phys. Rev. Lett. 80, 4305 (1998).

${ }^{8}$ I.I. Mazin, D.J. Singh, and C. Ambrosch-Draxl, Phys. Rev. B 59, 411 (1999).

${ }^{9}$ S. Ishibashi, T. Namikawa, and M. Satou, Jpn. J. Appl. Phys. 17, 249 (1978).

${ }^{10}$ H. Brändle, D. Weller, S.S.P. Parkin, J.C. Scott, P. Fumagalli, W. Reim, R.J. Gambino, R. Ruf, and G. Güntherodt, Phys. Rev. B 46, 13889 (1992).
${ }^{11}$ T. Tsujioka, T. Mizokawa, J. Okamoto, A. Fujimori, M. Nohara, H. Takagi, K. Yamaura, and M. Takano, Phys. Rev. B 56, R15 509 (1997).

${ }^{12}$ R.J. Soulen, Jr., J.M. Byers, M.S. Osofsky, B. Nadgorny, T. Ambrose, S.F. Cheng, P.R. Broussard, C.T. Tanaka, J. Nowak, J.S. Moodera, A. Barry, and J.M.D. Coey, Science 282, 85 (1998).

${ }^{13}$ E.J. Singley, C.P. Weber, D.N. Basov, A. Barry, and J.M.D. Coey, Phys. Rev. B 60, 4126 (1999).

${ }^{14}$ M.N. Iliev, A.P. Litvinchuk, H.-G. Lee, C.W. Chu, A. Barry, and J.M.D. Coey, Phys. Rev. B 60, 33 (1999).

${ }^{15}$ L. Ranno, A. Barry, and J.M.D. Coey, J. Appl. Phys. 81, 5774 (1997).

${ }^{16}$ A. Barry, J.M.D. Coey, L. Ranno, and K. Ounadjela, J. Appl. Phys. 83, 7166 (1998).

${ }^{17}$ K. Suzuki and P.M. Tedrow, Phys. Rev. B 58, 11597 (1998).

${ }^{18}$ X.W. Li, A. Gupta, T.R. McQuire, P.R. Duncombe, and G. Xiao, J. Appl. Phys. 85, 5585 (1999).

${ }^{19}$ M. Jaime, P. Lin, M.B. Salamon, and P.D. Han, Phys. Rev. B 58, R5901 (1998).

${ }^{20}$ A.J. Arko, J.A. Marcus, and W.A. Reed, Phys. Rev. 176, 671 (1968). 
${ }^{21}$ R.A. Young, J. Ruvalds, and L.M. Falicov, Phys. Rev. 178, 1043 (1969).

${ }^{22}$ R. Lück, Phys. Status Solidi 18, 49 (1966).

${ }^{23}$ N.W. Ashcroft, Phys. Kondens. Mater. 9, 45 (1969).

${ }^{24}$ J.-P. Jan, N.L. Martin, and A. Wenger, Phys. Rev. B 9, 1377 (1974).

${ }^{25}$ R.G. Chambers, Proc. Phys. Soc., London, Sect. A 65, 903 (1952).

${ }^{26}$ L. Berger and G. Bergmann, in Hall Effect and its Applications. Proceedings of the Commemorative Symposium, edited by C. L. Chien and C. R. Westgate (Plenum, New York, 1980), pp. 5576.

${ }^{27}$ C. M. Hurd, in Hall Effect and its Applications. Proceedings of the Commemorative Symposium (Ref. 26), pp. 1-54.

${ }^{28}$ M.J. Otto, H. Feil, R.A.M. van Woerden, J. Wijngaard, P.J. van der Valk, C.F. van Bruggen, and C. Haas, J. Magn. Magn. Mater. 70, 33 (1987).

${ }^{29}$ R. Asomoza, A. Fert, and R. Reich, J. Less-Common Met. 90, 177 (1983).

${ }^{30}$ A. B. Pippard, Magnetoresistance in Metals (Cambridge Univer- sity Press, Cambridge, 1988).

${ }^{31}$ C.B. Stagarescu, X. Su, D.E. Eastman, K.N. Altmann, F.J. Himpsel, and A. Gupta, Phys. Rev. B 61, R9233 (2000).

${ }^{32}$ S. Suzuki and M. Tomita, Jpn. J. Appl. Phys., Part 1 36, 4341 (1997).

${ }^{33}$ M.J. Otto, R.A.M. van Woerden, P.J. van der Valk, J. Wijngaard, C.F. van Bruggen, and C. Haas, J. Phys.: Condens. Matter 1, 2351 (1989).

${ }^{34}$ D.J. Sellmyer, J. Phys. Chem. Solids 30, 2371 (1969).

${ }^{35}$ K. Kubo and N. Ohata, J. Phys. Soc. Jpn. 33, 21 (1972).

${ }^{36}$ X.W. Li, A. Gupta, and G. Xiao, Appl. Phys. Lett. 75, 713 (1999).

${ }^{37}$ A.H. MacDonald, T. Jungwirth, and M. Kasner, Phys. Rev. Lett. 81, 705 (1998).

${ }^{38}$ X. Wang and X.-G. Zhang, Phys. Rev. Lett. 82, 4276 (1999).

${ }^{39}$ D.S. Chapin, J.A. Kafalas, and J.M. Honig, J. Phys. Chem. 69, 1402 (1965).

${ }^{40}$ R. V. Coleman, R. W. Klaffky, and W. H. Lowrey, in Hall Effect and its Applications. Proceedings of the Commemorative Symposium (Ref. 26), pp. 99-136. 